\title{
Perfil epidemiológico dos acidentes por animais peçonhentos na Bahia de 2010 a 2019
}

\author{
Epidemiological profile of accidents by poisonous animals in Bahia from 2010 to 2019 \\ Perfil epidemiológico de accidentes por animales venenosos em Bahía de 2010 a 2019
}

Recebido: 12/06/2021 | Revisado: 19/06/2021 | Aceito: 02/07/2021 | Publicado: 14/07/2021

\author{
Vitoria Vilas Boas da Silva Bomfim \\ ORCID: https://orcid.org/0000-0003-4897-0279 \\ Centro Universitário Jorge Amado, Brasil \\ E-mail: pesquisaclinica9@gmail.com \\ Renata Lisboa Santana \\ ORCID: https://orcid.org/0000-0002-9161-1987 \\ Universidade Salvador, Brasil \\ E-mail:renatahlisboa@hotmail.com \\ Claudia Danielle Guimarães \\ ORCID: https://orcid.org/0000-0002-2486-903X \\ Faculdade Integrada Cete, Brasil \\ E-mail: dani.36bernardo@gmail.com
}

\begin{abstract}
Resumo
Introdução: Os acidentes por animais peçonhentos são considerados um problema de saúde pública no Brasil. Atualmente, ocorrem cerca de 37 mil acidentes com escopiões, 26 mil acidentes com cobras e 20 mil com aranhas no país. Os dados relacionados a essas lesões são comunicados no sistema de informação de agravos de notificação. No entanto, faltam dados na literatura sobre os acidentes ocorridos no Nordeste, principalmente na Bahia. Objetivo: Descrever os aspectos epidemiológicos dos acidentes com animais peçonhentos no estado da Bahia entre os anos de 2010 a 2019, notificados no SINAN. Metodologia: Trata-se de um estudo observacionbal descritivo. Dados sobre os acidentes com animais peçonhentos na Bahia entre 2010 e 2019 foram coletados no SINAN. Resultados: Entre 2010 e 2019, foram registrados no SINAN 173.334 casos de acidentes por animais peçonhentos ocorridos na Bahia. Desyes, $70,66 \%$ foram causados por escorpiões, $15,07 \%$ por cobras, $3,58 \%$ por aranhas e 5,32\% por largatas e abelhas. Em $2,69 \%$ dos casos, não houve especificação dos agentes agressores. Considerações Finais: $\mathrm{O}$ presente estudo identificou que o perfil epidemiológico dos acidentes por animais peçonhentos atinge mais adultos jovens de 20 a 39 anos, faixa etária economicamente ativa e do sexo masculino, em sua maioria, evoluíram para cura apóso acidente com o animal.
\end{abstract}

Palavras-chave: Animais venenosos; Moderdura de serpentes; Venenos de escorpião; Venenos de aranha; Epidemiologia.

\begin{abstract}
Introduction: Accidents caused by venomous animals are considered a public health problem in Brazil. Currently, there are about 37 thousand accidents with scuppers, 26 thousand accidents with snakes and 20 thousand with spiders in the country. Data related to these injuries are communicated in the notification health information system. However, there is a lack of data in the literature on accidents that occurred in the Northeast, mainly in Bahia. Objective: To describe the epidemiological aspects of accidents with venomous animals in the state of Bahia between 2010 and 2019, reported in SINAN. Methodology: This is a descriptive observational study. Data on accidents with venomous animals in Bahia between 2010 and 2019 were collected from SINAN. Results: Between 2010 and 2019, 173,334 cases of accidents with venomous animals that occurred in Bahia were registered in the SINAN. Desyes, $70.66 \%$ were caused by scorpions, $15.07 \%$ by snakes, $3.58 \%$ by spiders and $5.32 \%$ by larks and bees. In $2.69 \%$ of cases, there was no specification of the offending agents. Final Considerations: The present study identified that the epidemiological profile of accidents by venomous animals affects more young adults aged 20 to 39 years, economically active age group and males, mostly, evolved to cure after an accident with the animal.
\end{abstract}

Keywords: Animals poisonous; Snake bites; Scorpion venoms; Spider venoms; Epidemiology.

\section{Resumen}

Introducción: Los accidentes causados por animales venenosos son considerados un problema de salud pública en Brasil. Actualmente, hay cerca de 37 mil accidentes con imbornales, 26 mil accidentes con serpientes y 20 mil con arañas en el país. Los datos relacionados con estas lesiones se comunican en el sistema de notificación de información sanitaria. Sin embargo, faltan datos en la literatura sobre accidentes ocurridos en el Nordeste, especialmente en Bahía. Objetivo: Describir los aspectos epidemiológicos de los accidentes con animales venenosos en el estado de Bahía entre 2010 y 2019, reportados en SINAN. Metodología: Se trata de un estudio observacional descriptivo. Los datos 
sobre accidentes con animales venenosos en Bahía entre 2010 y 2019 fueron recolectados del SINAN. Resultados: Entre 2010 y 2019 se registraron en el SINAN 173,334 casos de accidentes con animales venenosos ocurridos en Bahía. Desyes, $70,66 \%$ fueron causadas por escorpiones, $15,07 \%$ por serpientes, $3,58 \%$ por arañas y $5,32 \%$ por alondras y abejas. En el 2,69\% de los casos, no se especificó a los agentes infractores. Consideraciones Finales: El presente estudio identificó que el perfil epidemiológico de los accidentes por animales venenosos afecta a más adultos jóvenes de 20 a 39 años, grupo etario económicamente activo y machos, en su mayoría, evolucionados para curar tras un accidente con el animal.

Palabras clave: Animales venenosos; Mordedura de serpiente; Venenos de escorpio; Venenos de araña; Epidemiología.

\section{Introdução}

O Brasil tem uma fauna abundante, incluindo diversos animais de interesse médico, que podem causar danos à saúde humana. Dentro desse grupo, destacam-se os animais peçonhentos. Os acidentes compreendem envenenamentos causados por toxinas instiladas através dos aparelhos inoculadores, principalmente de serpentes (presas), escorpiões (ferrão) e aranhas (quelíceras), podendo determinar alterações locais e sistêmicas e até mesmo causar a morte. No Brasil, são registrados anualmente cerca de 37.000 acidentes por escorpiões, 26.000 por serpentes e 20.000 por aranhas (Brasil, 2014; Brasil, 2009).

Trata-se de um problema de Saúde Pública no Brasil de longa data, tanto pela frequência em que tais acidentes ocorrem, quanto pela gravidade que oferecem, podendo levar à morte ou promover sequelas capazes de gerar incapacidade temporária ou definitiva. Os estudos brasileiros na área iniciaram-se no campo do ofidismo, com os trabalhos desenvolvidos no início do século XX por Vital Brazil, até a década de 80, na região Sudeste (Vieira et al., 2009). Somente em junho de 1986, em decorrência da crise de produção de soro no país, foi implantado o Programa Nacional de Ofidismo, na Secretaria Nacional de Ações Básicas em Saúde do Ministério da Saúde (SNABS/MS), dando início a uma nova etapa no controle dos acidentes por animais peçonhentos. Nessa época, os acidentes ofídicos passaram a ser de notificação obrigatória no Brasil e dados sobre escorpionismo e araneísmo também começaram a ser coletados (Cardoso, 1993).

Atualmente, os registros desses acidentes ocorrem através do Sistema de Informação de Agravos de Notificação (SINAN/DATASUS/MS), do Sistema Nacional de Informações Tóxico-Farmacológicas (SINITOX/FIOCRUZ/MS), do Sistema de Informações Hospitalares (SIH/DATASUS/MS) e do Sistema de Informações sobre Mortalidade (SIM/DATASUS/MS) (Vieira et al., 2009). Permite-se, assim, uma relação de troca de informações para subsidiar a produção e distribuição dos diferentes tipos de soros, o mapeamento desses animais, programas de controle e prevenção de casos e agregar conhecimento aos profissionais que lidam com as vítimas, visto que a literatura nacional sobre o tema é escassa (dos Santos et al., 2012).

A rede de informações criada dessa forma garante conhecimento de grande relevância para a prática clínica, embasado o diagnóstico do tipo do envenenamento. Uma vez que a identificação do animal é pouco frequente, diagnostica-se o tipo de acidente por animal peçonhento através de informações epidemiológicas, sobretudo sua distribuição geográfica, e do quadro clínico apresentado pelo paciente (Brasil, 2009). Portanto, é fundamental o conhecimento das espécies de animais de importância médica e sua distribuição no território brasileiro, para que as devidas medidas preventivas e terapêuticas sejam tomadas.

O Brasil apresenta diversos gêneros de serpentes; dentre estes, as consideradas peçonhentas: Crotalus (Cascavel), Bothrops (Jararaca), Lachesis (Surucucu) e Micrurus (Corais Verdadeiras) (Vieira et al., 2009). Já os escorpiões de importância médica para o Brasil pertencem ao gênero Tityus, sobretudo as espécies Tityus Serrulatus (Escorpião Amarelo), Tityus Bahiensis (Escorpião Marrom), Tityus Stigmurus (Escorpião do Nordeste), Tityus Paraensis (Escorpião Preto), Tityus Metuendus (Escorpião Paraense). As aranhas de interesse médico no país, por sua vez, são representadas pelos gêneros Loxosceles (Aranha Marrom), Phoneutria (Armadeira) e Latrodectus (Viúva Negra) (Brasil, 2009). 
Apesar da existência de todos estes sistemas de informações oficiais e da relevância do assunto, os dados disponíveis não retratam a real magnitude do problema, provavelmente devido à subnotificação dos casos, graças, sobretudo, à dificuldade de acesso aos serviços de saúde em muitos municípios brasileiros (Vieira et al., 2009). Isso é aplicável, especialmente, nas regiões Norte e Nordeste, nas quais as características epidemiológicas de tais acidentes são pouco conhecidas, ainda que sua incidência seja elevada (Mise et al., 2007). Na Bahia, por exemplo, os acidentes por animais peçonhentos totalizam a maior parte dos casos registrados de envenenamentos, de acordo com o Centro Antiveneno da Bahia (CIAVE) (CIAVE, 2020).

Diante do exposto, o objetivo deste estudo é descrever os aspectos epidemiológicos dos acidentes por animais peçonhentos ocorridos no estado da Bahia, notificados no SINAN.

\section{Metodologia}

Trata-se de um estudo ecológico observacional e descritivo, incluindo todos os casos notificados como acidentes por animais peçonhentos envolvendo serpentes, aranhas, escorpiões, lagartas e abelhas ocorridos no estado da Bahia entre $2010 \mathrm{e}$ 2019 (Estrela, 2018). A coleta de dados relativos às notificações foi realizada em maio de 2020 no site do SINAN/DATASUS e foram incluídas as notificações incluídas até 31 de dezembro de 2019 (SESAB, 2020). A última atualização do SINAN havia sido realizada no dia 21 de janeiro de 2020. As variáveis extraídas e analisadas foram faixa etária, etnia, ano do acidente, gravidade (leve, moderado ou grave), desfechos (óbito pelo acidente, óbito por outra causa ou cura), microrregião de ocorrência, gênero do animal peçonhento envolvido e tempo entre a picada e o atendimento hospitalar. Os dados sobre a população total da Bahia, por sexo e faixa etária, foram coletados no site do Instituto Brasileiro de Geografia e Estatística (IBGE) para os anos de 2010 a 2020 (IBGE, 2013). Os referentes à população de cada microrregião foram coletados no site do DATASUS para os anos de 2010 a 2012 (DATASUS, 2021). Para os anos de 2013 a 2019, foram utilizados os dados do IBGE, publicados no Diário Oficial da União, referentes à população de cada município, agrupados posteriormente por microrregião (IBGE, 2019; IBGE, 2018; IBGE, 2017; IBGE, 2016; IBGE, 2015; IBGE, 2014; IBGE, 2013b). A partir dos dados obtidos, foram calculadas a taxa de incidência de acidentes na Bahia, a taxa de incidência média anual por microrregião por 100.000 pessoas e a letalidade dos acidentes. Os dados utilizados no estudo foram obtidos a partir de um banco de dados de acesso público e irrestrito, o que justifica a ausência do parecer do Comitê de Ética em Pesquisa (CEP).

\section{Resultados}

A Bahia é o estado com maior número absoluto de ocorrências e notificações de acidentes por animais peçonhentos do Nordeste e aparece em quarto lugar no cenário nacional, atrás de Minas Gerais, São Paulo e Paraná, respectivamente. No período compreendido entre 2010 a 2019, foram notificados no SINAN 173.334 casos de acidentes por animais peçonhentos ocorridos no estado da Bahia. Considerando a população da Bahia projetada pelo IBGE dos anos entre 2010 a 2019, a Bahia apresentou incidência média anual de 98 casos por 100 mil habitantes (IBGE, 2013). A Tabela 1 apresenta o número de acidentes por tipo de animal. Destes, 70,66\% foram causados por escorpiões, 15,07\% por serpentes, 3,58\% por aranhas e $5,32 \%$ por lagartas e abelhas. Em 2,69\% dos casos não havia especificação dos agentes agressores. 
Tabela 1 - Número de acidentes por tipo de animal peçonhento ocorridos na Bahia, notificados pelo SINAN, no período de 2010 a 2019. Brasil, 2021.

\begin{tabular}{ccccccc}
\hline ANO & ESCORPĨ̃O & SERPENTE & ARANHA & OUTROS* & IGN/BRANCO & $\begin{array}{c}\text { NÃO } \\
\text { ESPECÍFICADO }\end{array}$ \\
\hline $\mathbf{2 0 1 0}$ & 8925 & 3055 & 422 & 550 & 463 & 239 \\
$\mathbf{2 0 1 1}$ & 10335 & 3158 & 501 & 689 & 544 & 280 \\
$\mathbf{2 0 1 2}$ & 9179 & 2757 & 498 & 596 & 486 & 258 \\
$\mathbf{2 0 1 3}$ & 10834 & 2645 & 594 & 598 & 647 & 344 \\
$\mathbf{2 0 1 4}$ & 11881 & 2240 & 512 & 902 & 690 & 396 \\
$\mathbf{2 0 1 5}$ & 10159 & 2516 & 482 & 1023 & 311 & 436 \\
$\mathbf{2 0 1 6}$ & 9323 & 2542 & 531 & 821 & 252 & 395 \\
$\mathbf{2 0 1 7}$ & 14135 & 2625 & 854 & 944 & 407 & 598 \\
$\mathbf{2 0 1 8}$ & 19093 & 2251 & 978 & 1399 & 473 & 853 \\
$\mathbf{2 0 1 9}$ & 18608 & 2336 & 843 & 1704 & 398 & 806 \\
\hline TOTAL & $\mathbf{1 2 2 4 9 2}$ & $\mathbf{2 6 1 2 5}$ & $\mathbf{6 2 1 5}$ & $\mathbf{9 2 2 6}$ & $\mathbf{4 6 7 1}$ & $\mathbf{4 6 0 4}$ \\
\hline
\end{tabular}

*Lagartas e Abelhas. Fonte: Autores (2021).

Os acidentes ofídicos notificados no período foram causados por serpentes peçonhentas dos gêneros Crotalus (0,82\%), Bothrops (10,78\%), Lachesis (0,07\%), Micrurus ( $0,17 \%)$ e serpentes não peçonhentas $(0,62 \%)$. Em 87,55\% dos casos o gênero da serpente não foi identificado (Gráfico 1).

Gráfico 1 - Número total de notificações por gênero da serpente no período de 2010 a 2019. Brasil, 2021.

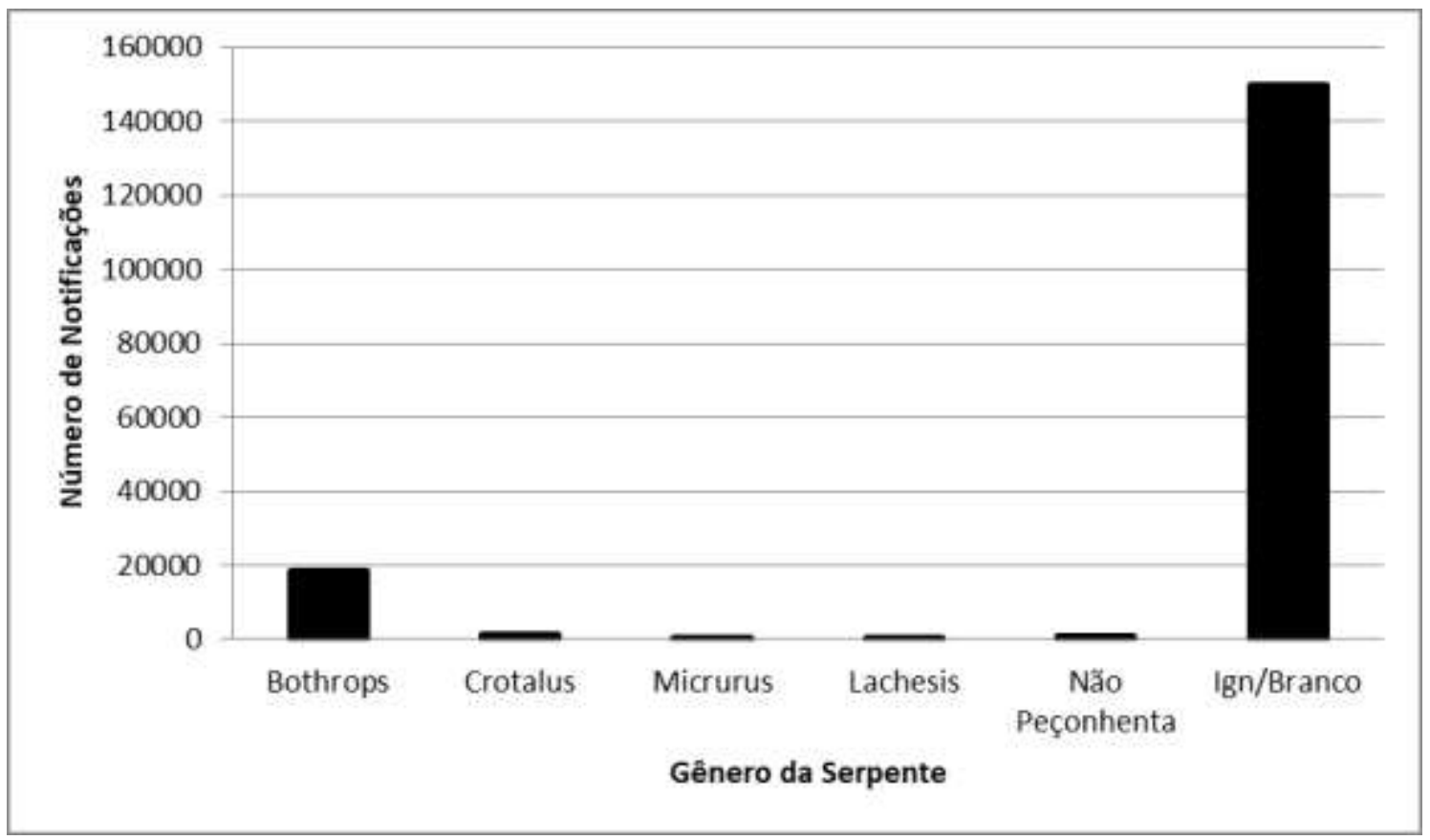

Fonte: Autores (2021).

Quanto aos acidentes aracnídicos, não se pôde identificar os agentes agressores em 98,76\% dos casos. Os acidentes em que houve identificação do agente foram provocados por aranhas dos gêneros Loxosceles $(0,15 \%)$, Phoneutria $(0,30 \%)$ e Latrodectus $(0,09 \%)$ e por outras espécies $(0,68 \%)$ (Gráfico 2). No período entre 2010 e 2019, a incidência média de acidentes 
por escorpiões correspondeu a 68 casos/100.000 habitantes/ano, por serpentes 18 casos/100.000 habitantes/ano e por aranhas 3 casos/100.000 habitantes/ano.

Gráfico 2 - Número total de notificações por gênero da aranha no período de 2010 a 2019. Brasil, 2021.

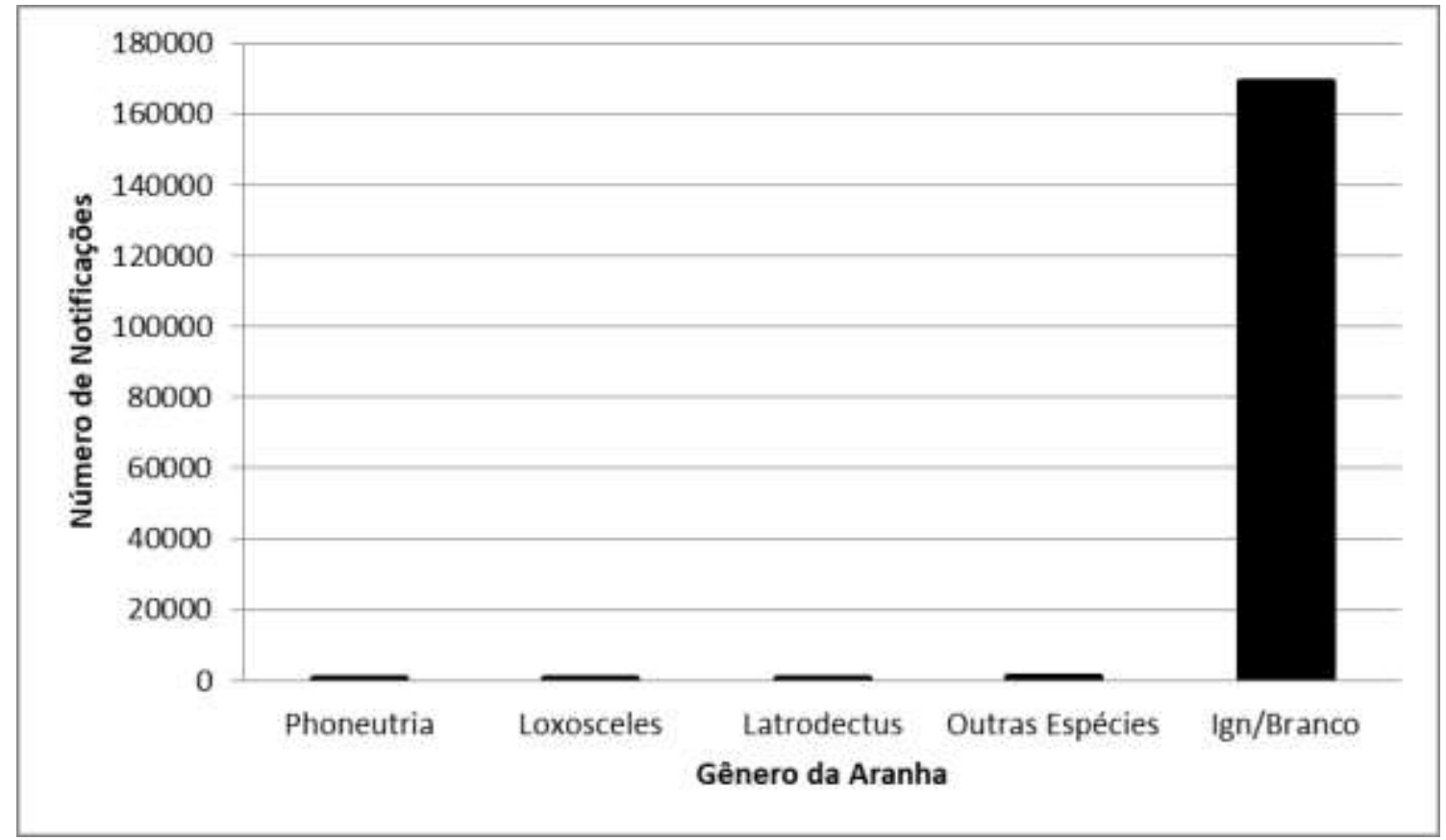

Fonte: Autores (2021).

A população acometida por raça branca foi de $10,6 \%, 10,22 \%$ de raça preta, $60,51 \%$ possuiam a raça parda, $0,80 \%$ de raça amarela, 0,55\% eram de raça indigena. Em 17,29\% dos casos foi ignorado a raça. Os acidentes foram mais frequentes no sexo masculino (53,7\%). Com relação à faixa etária, observou-se maior frequência de acidentes entre 20 e 39 anos, contribuindo para $32,3 \%$ das ocorrências.

As microrregiões do estado da Bahia com maior número absoluto de notificações de acidentes por animais peçonhentos foram Jequié, com total de 7918 casos notificados no período, e Feira de Santana, com total de 4609. A Figura 1 representa a distribuição geográfica das notificações de acidentes por animais peçonhentos no estado da Bahia no período entre 2010 e 2019. 
Figura 1 - Distribuição geográfica das notificações por microrregião da Bahia entre 2010 e 2019. Brasil, 2021.

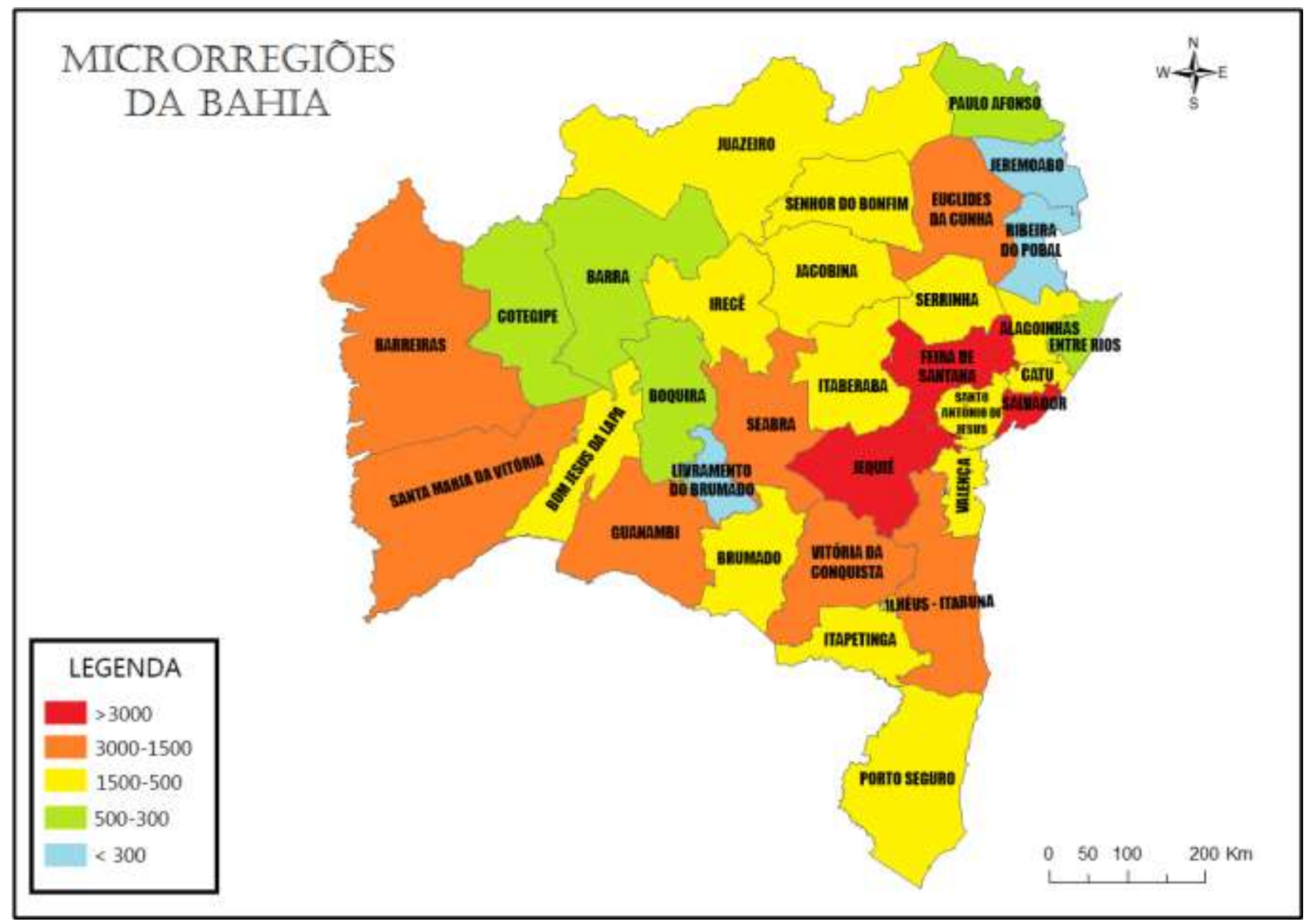

Fonte: Autores (2021).

O tempo decorrido entre o momento da picada e o atendimento médico foi, predominantemente, menor que 1 hora (43,98\%). Em 27,24\% dos casos, o atendimento ocorreu entre 1 e 3 horas após a picada, em 7,9\% ocorreu entre 3 e 6 horas e em 9,24\% após 6 horas. O tempo decorrido não foi notificado em 11,63\% dos acidentes, como mostra no Gráfico 3. 
Gráfico 3 - Tempo decorrido entre o momento do acidente e o atendimento em uma unidade de saúde. Brasil, 2021.

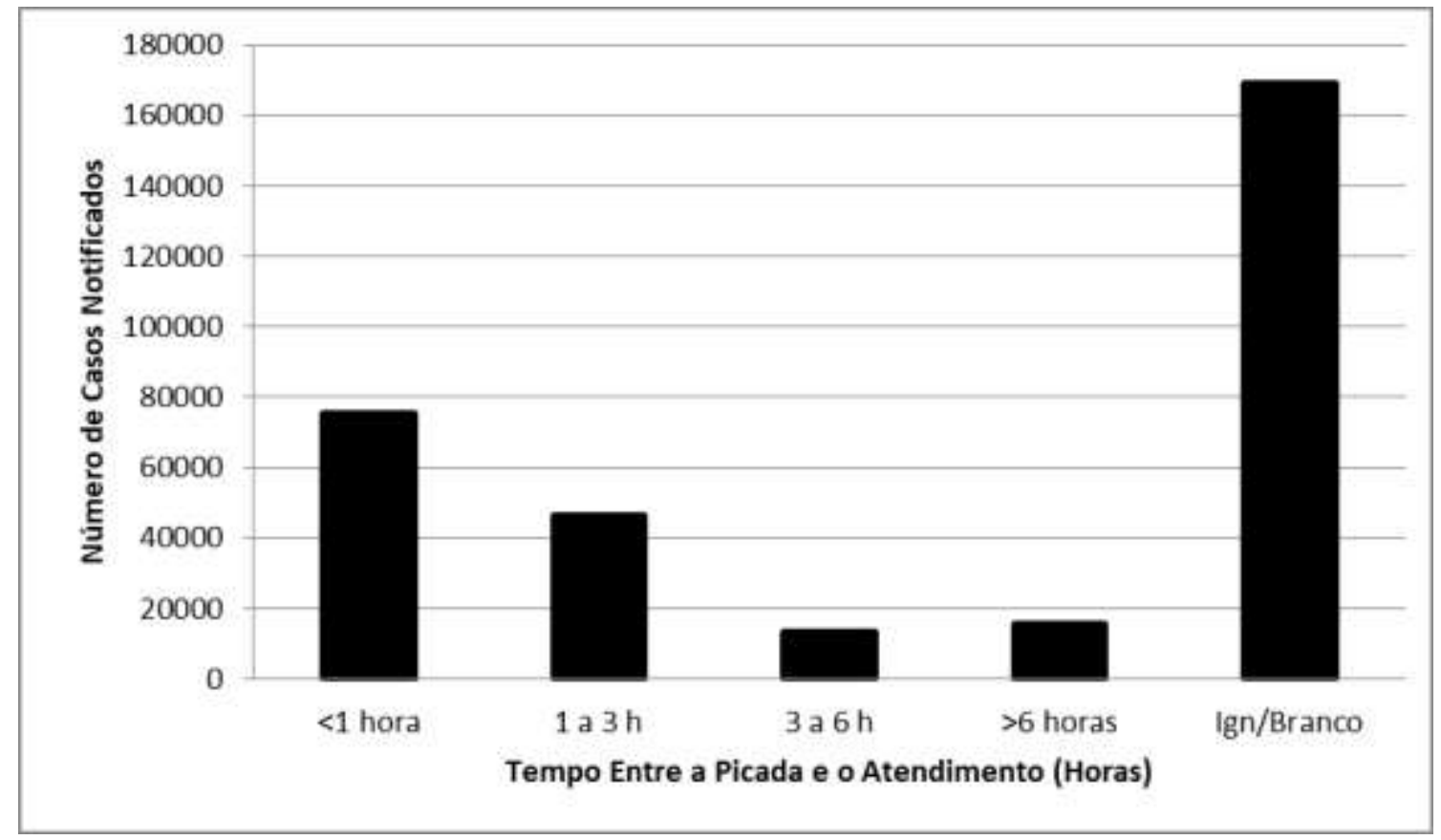

Fonte: Autores (2021).

Quanto à gravidade dos acidentes em geral, 78,84 \% dos casos foram considerados leves, 13,35\% moderados, 1,76\% graves e, em 6,03 \% dos casos a gravidade não foi especificada. Os acidentes escorpiônicos 84,55\% dos casos foram classificados como leves, em torno de 9,72 \% foram moderados e 0,88 \% graves. Em relação aos acidentes aracnídeos, os casos com gravidade leve correspondem a $81,45 \%$, os de gravidade moderada a $10,48 \%$ e os casos graves totalizaram $0,82 \%$. Já os acidentes ofídicos, os casos com gravidade leve correspondem a 50,50\%, os de gravidade moderada a 34,17\% e os casos graves totalizaram 6,86\%. No restante dos casos (aproximadamente 9,9\%), a gravidade não foi informada.

Dos 173.334 casos notificados, 87,78 \% evoluíram para a cura, 0,23\% evoluíram para óbito por outra causa, enquanto em $11,96 \%$ dos casos a evolução não foi notificada e em $0,02 \%$ dos casos ocorreu o óbito pelo agravo notificado. Os acidentes por escorpiões que apresentaram letalidade foram de aproximadamente $13,8 \%$ e aranhas apresentaram letalidade de aproximadamente $0,8 \%$, enquanto os acidentes por serpentes tiveram letalidade na faixa de $6,8 \%$.

\section{Discussão}

A Bahia é o estado com o maior número de ocorrências e notificações de acidentes por animais peçonhentos do Nordeste e aparece em quarto lugar no cenário nacional, atrás de Minas Gerais, São Paulo e Paraná, respectivamente. Podemos citar como alguns fatores de risco determinantes, condições sociais como saneamento básico, desmatamento, educação, acesso aos bens e serviços em geral, entre outros (SESAB, 2019).

O sexo masculino apresenta-se com maior prevalência de vulnerabilidade aos ataques por animais peçonhentos quando comparado à pesquisa feminina. Estudos evidenciam que a prevalência do sexo masculino é justificado por ser um número maior de trabalhadores braçais e civis no mercado tornam-se alvos deste tipo de acidente, e que o ambiente que ocorrem estes ataques no alvo feminino é na própria residência (da Silva et al., 2017). 
A faixa etária de pacientes envolvidos em acidentes por animais peçonhentos é mais frequente entre 20 a 39 anos. Os casos notificados por animais peçonhentos nas microrregiões do estado da bahia, destacam-se as cidades de Jequié e Feira de Santana (SESAB, 2019; DATASUS, 2021).

A maioria dos acidentes registrados no período quanto a sua gravidade em geral foram considerados leves, levando a cura, que pode está associado ao curto intervalo entre a picada e o atendimento, como já foi evidenciado na literatura, a rapidez no atendimento está diretamente associada à evolução do acidente (Evangelista \& Azevedo, 2016).

O perfil epidemiologico mais acometido são pessoas do sexo masculino adultos jovem entre 20-39 anos de raça parda na região de Jequie, o animal peçonhento que mais registrou caso foi os escorpiões e os atendimentos em sua maioria foram em até 1 hora da picada do animal (SESAB, 2019; DATASUS, 2021).

Por outro lado, conhecer o perfil epidemiológico dos acidentes escorpiônicos através de estudo com metodologias analíticas, é recomendado, uma vez as limitações inerentes a um estudo descritivo são bem descritas na literatura. Além disso, as limitações deste estudo podem estar relacionadas ao uso dos dados do SINAN, e uma possível diferença em relação aos dados do Centro de Informações Antiveneno da Bahia, da Secretaria de Saúde do Estado (Brasil, 2021).

É possível, ainda, a ocorrência de sub-notificação. Ainda assim, é necessário especial atenção dos gestores do Sistema Único de Saúde às microrregiões evidenciadas neste estudo, revelada notadamente nos acidentes graves que podem deixar sequelas bem como nos óbitos que são plenamente evitáveis (Brasil, 2021).

\section{Considerações Finais}

Os casos de acidentes por animais peçonhentos na Bahia, seguem o perfil nacional com casos atingindo em sua maioria o sexo masculino, em idade economicamente ativa, comum no período entre o fim das chuvas e início da seca e maior incidência de acidentes com escorpiões, seguido por serpentes e aranhas. A diferença reside na ausência de notificações de acidentes por outras causas como os himenópteros. Ainda existe a subnotificação nos acidentes por abelhas, marimbondos e vespas, possivelmente em decorrência da ausência de soro específico para tratamento, como a terapêutica envolve apenas ações inespecíficas e direcionadas ao suporte clínico às reações apresentadas pelo paciente, as notificações não são tão efetivas como as que o soro é fornecido mediante sua efetivação, impossibilitando assim dimensionar a gravidade desses casos.

Em razão da falta de soro no país, o controle rigoroso das ampolas fica exclusivamente com a Vigilância Epidemiológica para que todos os casos sejam criteriosamente avaliados e acompanhados, no município não houve necessidade de transferir pacientes para outros locais por falta do soro, porém o estoque é mínimo e muitas vezes houve necessidade de buscar com urgência em municípios vizinhos para administrar nos acidentados por animais peçonhentos, ampliando assim o tempo de início da soroterapia. Esse estudo contribui em três aspectos: a importância do Enfermeiro, profissional indispensável, que tem a responsabilidade de identificar os agravos e providenciar os soros necessários para atender as vítimas de animais peçonhentos mais frequentes na região que atua, assim impactando na redução da subnotificação. E na Atenção Primária, primeiro lugar procurado pela vítima, contribuindo para prevenção da deterioração clínica e óbito. Ficou evidenciado, que é necessário que políticas públicas definam estratégias que dêem suporte para esta realidade presente em nosso estado.

\section{Referências}

Brasil. Ministério da Saúde. (2009). Secretaria de Vigilância em Saúde. Departamento de Vigilância Epidemiológica. Guia de Vigilância Epidemiológica.

Brasil. Ministério da Saúde. (2014). Secretaria de Vigilância em Saúde. Departamento de Vigilância Epidemiológica. Acidentes por Animais Peçonhentos. In:Guia de vigilância em Saúde. 1st ed. 
Research, Society and Development, v. 10, n. 8, e38710817113, 2021

(CC BY 4.0) | ISSN 2525-3409 | DOI: http://dx.doi.org/10.33448/rsd-v10i8.17113

Brasil. Ministério da Saúde. (2021). Secretaria de Vigilância em Saúde. Coordenação Geral de Desenvolvimento da Epidemiologia em Serviços. Guia de Vigilância em Saúde: volume único.

Cardoso, J. L. C. (1993). Acidentes por animais peçonhentos na Coordenação de Zoonoses e Animais Peçonhentos-comentários e sugestões. Brasília: Ministério da Saúde.

Centro Antiveneno da Bahia (CIAVE). (2020). Controle de Acidentes por Animais Peçonhentos. http://www.saude.ba.gov.br/ciave/index.php?option=com_contente\&view=article\&id=349\&Itemid=23.

da Silva, P. L. N., de Andrade Costa, A., Damasceno, R. F., de Oliveira Neta, A. I., Ferreira, I. R., \& Fonseca, A. D. G. (2017). Perfil epidemiológico dos acidentes por animais peçonhentos notificados no Estado de Minas Gerais durante o período de 2010-2015. Revista Sustinere, 5(2), 199-217.

DATASUS. (2021). População residente - Bahia. http://tabnet.datasus.gov.br/vgi/tabcgi.exe?ibge/cnv/popba.def.

dos Santos, J. M., da Silva Croesy, G., \& Marinho, L. F. B. (2012). Perfil epidemiológico dos acidentes escorpiônicos em crianças, no estado da Bahia, de 2007 a 2010. Revista Enfermagem Contemporânea, 1(1).

Estrela, C. (2018). Metodologia Científica: Ciência, Ensino, Pesquisa. Editora Artes Médicas.

Evangelista, G. F., \& Azevedo, C. S. D. (2016). Arachnidism, scorpionism and ophidism in Ouro Preto Municipality, Minas Gerais State, Brazil. Revista da Sociedade Brasileira de Medicina Tropical, 49(6), 786-789.

Instituto Brasileiro de Geografia e Estatística (IBGE). (2013). Projeção da População do Brasil e Unidade da Federação por Sexo e Idade para o período de 2000-2030. http://www.ibge.gov.br/estadosat/temas.php?sigla=ba\&tema=projecao2013.

Instituto Brasileiro de Geografia e Estatística (IBGE). (2021). Estimativas populacionais para os municípios e para as Unidades da Federação brasileiros em 01.07.2019. http://www.ibge.gov.br/home/estatistica/população/estimativa2019/estimativa_dou.shtm.

Instituto Brasileiro de Geografia e Estatística (IBGE). (2021). Estimativas populacionais para os municípios e para as Unidades da Federação brasileiros em 01.07.2018. http://www.ibge.gov.br/home/estatistica/população/estimativa2018/estimativa_dou.shtm.

Instituto Brasileiro de Geografia e Estatística (IBGE). (2021). Estimativas populacionais para os municípios e para as Unidades da Federação brasileiros em 01.07.2017. http://www.ibge.gov.br/home/estatistica/população/estimativa2017/estimativa_dou.shtm.

Instituto Brasileiro de Geografia e Estatística (IBGE). (2021). Estimativas populacionais para os municípios e para as Unidades da Federação brasileiros em 01.07.2016. http://www.ibge.gov.br/home/estatistica/população/estimativa2016/estimativa_dou.shtm.

Instituto Brasileiro de Geografia e Estatística (IBGE). (2021). Estimativas populacionais para os municípios e para as Unidades da Federação brasileiros em 01.07.2015. http://www.ibge.gov.br/home/estatistica/população/estimativa2015/estimativa_dou.shtm.

Instituto Brasileiro de Geografia e Estatística (IBGE). (2021). Estimativas populacionais para os municípios e para as Unidades da Federação brasileiros em 01.07.2014. http://www.ibge.gov.br/home/estatistica/população/estimativa2014/estimativa_dou.shtm.

Instituto Brasileiro de Geografia e Estatística (IBGE). (2021). Estimativas populacionais para os municípios e para as Unidades da Federação brasileiros em 01.07.2013. http://www.ibge.gov.br/home/estatistica/população/estimativa2013/estimativa_dou.shtm.

Mise, Y. F., Lira-da-Silva, R. M., \& Carvalho, F. M. (2007). Envenenamento por serpentes do gênero Bothrops no Estado da Bahia: aspectos epidemiológicos e clínicos. Revista da Sociedade Brasileira de Medicina Tropical, 40(5), 569-573.

Secretaria da Saúde do Estado da Bahia (SESAB). (2020). Portal de Vigilancia da Saúde. http://www3.saude.ba.gov.br/cgi/tabcgi.exe?tabnet/sinan/animp.def.

Vieira, E. M., Perdona, G. D. C. S., Almeida, A. M. D., Nakano, A. M. S., Santos, M. A. D., Daltoso, D., \& Ferrante, F. G. D. (2009). Conhecimento e atitudes dos profissionais de saúde em relação à violência de gênero. Revista Brasileira de Epidemiologia, 12(4), 566-577. 